\title{
Introducción y desarrollo de variedades de algodón Upland en el sistema productivo colombiano: Una revisión
}

\section{Introduction and development of Upland cotton cultivars in the Colombian production system: A review}

Fecha de recepción: 10 de septiembre de 2017 Fecha de aprobación: 29 de diciembre de 2017
Oscar Burbano-Figueroa ${ }^{1}$

Karen Sofía Montes-Mercado ${ }^{2}$ Iván Javier Pastrana-Vargas ${ }^{3}$ Jorge Cadena-Torres ${ }^{4}$

DOI: https://doi.org/10.19053/01228420.v15.n1.2018.7754

\section{Resumen}

El propósito de esta revisión es ofrecer un documento comprensivo de los cultivares de algodón Upland introducidos en los sistemas de producción colombianos. En el caso de las variedades nacionales, toda la información referida a genealogía y características se encuentra dispersa en documentos de literatura gris, lo cual limita su hallazgo, acceso y evaluación. En este contexto, los objetivos de esta revisión fueron: i) describir las características y genealogía de las variedades de algodón Upland desarrolladas por los programas de mejoramiento en Colombia y ii) describir las variedades de algodón Upland introducidas desde otros países en el sistema productivo colombiano. La información aquí descrita es parte de un proceso de consolidación de datos dispersos en entidades públicas y privadas del cultivo de algodón desarrollado con fines de análisis de metadata. En el futuro, este documento puede ser usado como guía descriptiva para programas de mejoramiento genético, incorporación de nuevas variedades o evaluación del impacto del desarrollo e introducción de variedades sobre las brechas de rendimiento y productividad; adicional-

1 M.Sc. Corporación Colombiana de Investigación Agropecuaria - CORPOICA (Cereté-Córdoba, Colombia). ORCID: 0000-0002-6604-7333.

2 Corporación Colombiana de Investigación Agropecuaria-CORPOICA (Cereté-Córdoba, Colombia).kmontes@corpoica. org.co. ORCID: 0000-0002-3103-5981.

3 M.Sc. Corporación Colombiana de Investigación Agropecuaria - CORPOICA (Agustín Codazzi-Cesar, Colombia). pastrana@corpoica.org.co. ORCID: 0000-0002-8756-1083.

4 Ph.D. Corporación Colombiana de Investigación Agropecuaria - CORPOICA (Cereté-Córdoba, Colombia). jcadena@ corpoica.org.co. ORCID: 0000-0002-5180-2893. 
mente, incorpora una serie de recomendaciones para nuevas variedades, basados en el actual escenario que enfrenta el sector algodonero.

Palabras clave: Corpoica; Gossypium; ICA; LCER; líneas avanzadas de mejoramiento (LAM); mejoramiento genético.

\begin{abstract}
The purpose of this review was to provide a comprehensive description of the Upland cotton cultivars incorporated into the Colombian production systems. Genealogy and traits data of the Colombian cultivars is scattered across grey literature which reduces chances to discover, access and evaluate this information.Consequently, the objectives of this review were: i) to describe traits and genealogy of the Upland cotton cultivars developed by the breeding programs in Colombia, and ii) to describe the Upland cotton cultivars from other countries that have been introduced into the Colombian production system. This data repository is part of the efforts dedicated to consolidate data dispersed in public and private institutions with the purpose of metadata analysis. In the future, this document will provide guidance to procedures of breeding, introduccion of new varieties, and assessment of development and introduction of new cultivars in closing the yield gaps. Additionally, this review includes recommendations to develop new cultivars based on the current scenario of cotton producers.
\end{abstract}

Keywords: advanced breeding lines; Corpoica; Genetic breeding; Gossypium; ICA; LCER.

\title{
Para citar este artículo:
}

Burbano-Figueroa O., Montes-Mercado KS., Pastrana-Vargas IJ., Cadena-Torres J. Introducción y desarrollo de variedades de algodón Upland en el sistema productivo colombiano: Una revisión. Ciencia y Agricultura. 2018; 15(1): 29-44. 


\section{Introducción}

El género Gossypium, perteneciente a la familia Malvaceae y a la tribu Gossypieae, incluye 50 especies; cuatro de estas son cultivadas para obtener fibra de hilatura, y las otras 46 están distribuidas a través del trópico y el subtrópico en formas silvestres. Las especies silvestres de Gossypium son fuentes importantes de características genéticas: propiedades particulares de fibra, esterilidad masculina citoplasmática, resistencia a factores bióticos y abióticos y otras más. Las variedades cultivadas provienen de una base genética estrecha, y la introgresión se ha convertido en el principal método para incrementar la diversidad genética (1-3).

La especie alotetraploide Gossypium hirsutum de algodón es la más extendida en el mundo y, a la vez, la principal fuente de fibras naturales; además, es la planta poliploide preferida como modelo biológico. El género Gossypium se originó a partir de un eudicot progenitor hexaploide y la subsecuente diversificación en ocho grupos de genoma diploide, incluyendo los Ilamados grupos A-G y K (2). El genoma A diploide, proveniente de África, diverge del progenitor eudicot, junto al genoma D Mexicano, hace 5 10 millones de años. Estos dos genomas se unificaron hace unos dos millones de años. Un ancestro del genoma A, similar a la actual especie Gossypium arboreum, sufrió dispersión transoceánica, alcanzando el Nuevo Mundo e hibridizándose con un ancestro del genoma $\mathrm{D}$, similar a Cossypium raimondii. Esta hibridación, con posterior doblamiento de cromosomas, dio origen al algodón alotetraploide, que se dispersó desde las Américas hacia el Pacífico Occidental, originando varias especies; entre estas se encuentra G. hirsutum, que se conoce como algodón Upland, cuya alta productividad de campo ha hecho que represente hoy más del $90 \%$ del algodón cultivado a nivel mundial $(3,4)$.

El cultivo de algodón en Colombia, así como la producción de fibra de este, están reportados desde los años veinte y treinta del siglo pasado (5). Las explotaciones del algodón en Colombia se iniciaron en 1834, año desde el cual el cultivo empezó a tener repercusión económica nacional. En los años treinta ocurrieron hechos importantes, como el cambio de variedades perennes por variedades anuales (6). La producción mecanizada y comercial de algodón se estableció a finales de los años cuarenta, y las áreas de siembra mantuvieron un carácter expansivo hasta la década de los setenta $(7,8)$. La máxima área de siembra de algodón se alcanzó en 1977, con un total de 377.246 ha. En el marco de la economía nacional, el algodón llegó a ocupar el segundo lugar de exportación, después del café, hasta 1978, cuando, por diversos factores adversos, las áreas de siembra disminuyeron de manera constante hasta el año 1982, cuando solo se sembraron 68.377 ha. A partir de 1983, las áreas de siembra se incrementaron nuevamente, hasta alcanzar las 261.939 ha en 1991; pero en 1992 se reinicia un proceso de reducción hasta el nivel actual de menos de 10,000 ha (9). Esta disminución de las áreas de siembra es resultado de factores políticos, económicos y biofísicos, tales como la política de apertura económica en el país, el desmonte de los subsidios a la agricultura, la reducción en la protección arancelaria, las altas tasas de inflación e interés bancario, la caída de los precios internacionales de la fibra y el incremento de los costos de producción y de las brechas de rendimientos, asociado a variables climáticas y biológicas $(7,8,10)$. Sin embargo, el cultivo de algodón prevalece en regiones donde históricamente ha sido cultivado (Córdoba y Tolima) y en donde representa la actividad industrial de mayor relevancia y el mayor generador de empleo rural $(8,11)$.

El algodón Upland llegó a cultivarse en 25 de los 32 departamentos del país, divididos en dos temporadas de cultivo alternadas, que aún siguen vigentes. La denominada temporada "Costa-Meta" (TCM) incluye las siembras desarrolladas en los departamentos de Córdoba, Cesar, Sucre, Atlántico, Bolívar, Guajira y Magdalena, en las Planicies del Caribe, y en los departamentos de Casanare, Meta, Guaviare y Vichada, en los Llanos Orientales, durante la segunda mitad del año, entre agosto y octubre, y cosechadas entre los meses de diciembre y marzo. En el primer semestre se establecen los cultivos de la denominada "Temporada del interior" (TIR), en los departamentos de Caldas, Cauca, Cundinamarca, Huila, Risaralda, Tolima y Valle del Cauca; estos cultivos son sembrados entre abril y junio y cosechados entre julio y octubre de cada año (12). 
Actualmente, dada la reducción en las áreas de siembra, las siembras de algodón de la TCM se concentran en los departamentos de Córdoba y Cesar, mientras que las siembras de la TIR se concentran en el Tolima y Huila.

La introducción de materiales genéticos estadounidenses de siembra al mercado colombiano fue constante durante el siglo pasado. El algodón Upland fue introducido por primera vez en Colombia en el año 1935, con variedades de la serie DeltaPine (DP), pertenecientes a la colección de germoplasma de algodón mejorado de Estados Unidos (5); de allí en adelante, las variedades DP, junto a variedades desarrolladas internamente por el Programa de Mejoramiento Genético de Algodón en Colombia, dominaron el mercado de las semillas en Colombia (13). A partir de la década de los noventa, las variedades predominantes en Colombia corresponden a las de fibra de longitud media, desarrolladas en Colombia, USA y Australia. Delta Opal (DO) es la variedad de fibra media más representativa de este periodo (14).
La introducción de las variedades transgénicas a Colombia se da en el año 2003, con la introducción del cultivar DP NuOpal, también de origen americano, convirtiéndose en la primera variedad transgénica de algodón sembrada en el país. Esta variedad contenía la proteína Cry1Ac (Bollgard), que confería resistencia a lepidópteros plaga. A partir de este año, las variedades de origen americano incorporadas en el sistema productivo de algodón colombiano son del tipo transgénico (Tabla I) (11, 15-19). Actualmente, los cultivares transgénicos contienen una diversidad de genes que les confieren características como resistencia a insectos lepidópteros y tolerancia a diferentes tipos de herbicidas (Tabla II) (2025). Las series de cultivares transgénicos usados en la actualidad incluyen variedades designadas como Deltapine (DP), Stoneville (SV) y FiberMax (FM); estas dos últimas series de variedades han dominado el mercado colombiano durante la última década.

Tabla I. Descripción de tecnologías transgénicas disponibles en materiales de algodón introducidos en Colombia. Datos originales con descripción de resoluciones del ICA disponibles en (11, 15-19).

\begin{tabular}{|c|c|c|c|c|}
\hline Tecnología & $\begin{array}{c}\text { Genes } \\
\text { transgénicos }\end{array}$ & $\begin{array}{l}\text { Año de } \\
\text { liberación }\end{array}$ & Regiones aprobadas & $\begin{array}{l}\text { Cultivares de algodón que } \\
\text { contienen la tecnología }\end{array}$ \\
\hline \multirow[t]{2}{*}{ Bollgard } & \multirow[t]{2}{*}{ Cry1Ac } & 2003 & $\begin{array}{l}\text { Caribe Húmedo y Seco, } \\
\text { Tolima, Huila y Valle del } \\
\text { Cauca }\end{array}$ & \multirow[t]{2}{*}{ NuOpal Bollgard } \\
\hline & & 2007 & Llanos Orientales & \\
\hline \multirow[b]{2}{*}{ Roundup Ready } & \multirow[b]{2}{*}{ CP4 EPSPS } & 2004 & Caribe Húmedo y Seco & Delta Opal RR \\
\hline & & 2007 & $\begin{array}{l}\text { Alto Magdalena y Valle } \\
\text { del Cauca }\end{array}$ & \\
\hline \multirow{2}{*}{$\begin{array}{l}\text { Bollgard - } \\
\text { Roundup Ready }\end{array}$} & Cry1Ac & \multirow{2}{*}{2007} & \multirow{2}{*}{$\begin{array}{l}\text { Alto Magdalena, Valle } \\
\text { del Cauca, Caribe } \\
\text { Húmedo y Seco y Llanos } \\
\text { Orientales }\end{array}$} & \multirow{2}{*}{ DP455 BGRR } \\
\hline & CP4 EPSPS & & & \\
\hline \multirow{5}{*}{$\begin{array}{l}\text { Bollgard II - } \\
\text { Roundup Ready } \\
\text { Flex }\end{array}$} & Cry1Ac & \multirow{5}{*}{2007} & \multirow{5}{*}{$\begin{array}{l}\text { Alto Magdalena, Valle } \\
\text { del Cauca, Caribe } \\
\text { Húmedo y Seco y Llanos } \\
\text { Orientales }\end{array}$} & FM9063 B2F \\
\hline & Cry2Ab & & & FM9180 B2F \\
\hline & CP4 EPSPS (2) & & & FM9162 B2F \\
\hline & & & & FM9171 \\
\hline & & & & DP141 B2RF \\
\hline LI cotton & PAT & 2010 & Caribe Húmedo & $\begin{array}{l}\text { Cultivar no especificado en } \\
\text { resoluciones del ICA. No } \\
\text { información disponible en el } \\
\text { mercado }\end{array}$ \\
\hline
\end{tabular}


Tabla II. Descripción de genes transgénicos incorporados en los materiales de algodón liberados en el mercado (16-21).

\begin{tabular}{|c|c|c|c|c|}
\hline Gene transgénico & $\begin{array}{c}\text { Nombre } \\
\text { comercial }\end{array}$ & Propietario & Actividad biológica & Referencia \\
\hline \multicolumn{5}{|c|}{ Proteínas con actividad insecticida } \\
\hline cry $1 \mathrm{Ac}$ & Bollgard & Monsanto & Control de lepidópteros & $\begin{array}{l}\text { James 2003, Olsen et al. } \\
\text { 2005; Greenplate et al. } 2003\end{array}$ \\
\hline cry $1 \mathrm{Ac}+$ cry $2 \mathrm{Ab}$ & Bollgard II & Monsanto & Control de lepidópteros & Sivasupramaniam et al. 2008 \\
\hline cry $1 \mathrm{Ac}+$ cry $1 \mathrm{~F}$ & Widestrike & $\begin{array}{l}\text { Dow } \\
\text { Agrosciences }\end{array}$ & Control de lepidópteros & $\begin{array}{l}\text { Adamczyk y Gore, 2004; } \\
\text { Siebert et al., 2009; Tindall } \\
\text { et al. } 2009\end{array}$ \\
\hline Vip3A & VipCot & Syngenta & Control de lepidópteros & $\begin{array}{l}\text { Kurtz et al., 2007; } \\
\text { Bommireddy y Leonard, } \\
\text { 2008; Ali y Luttrell, } 2011\end{array}$ \\
\hline \multicolumn{5}{|c|}{ Proteína responsable de la resistencia a herbicidas } \\
\hline CP4 EPSPS & Roundup Ready & Monsanto & Resistencia a glifosato & Funke et al. 2006 \\
\hline CP4 EPSPS (2) & $\begin{array}{l}\text { Roundup Ready } \\
\text { Flex }\end{array}$ & Monsanto & Resistencia a glifosato & Que et al. 2010 \\
\hline PAT & LibertyLink & Bayer & $\begin{array}{l}\text { Resistencia a } \\
\text { glufosinato }\end{array}$ & Sweeney and Jones 2014 \\
\hline
\end{tabular}

El desarrollo de variedades nacionales de algodón ha estado a cargo de dos entidades; en primer lugar, durante los primeros años de desarrollo del cultivo el desarrollo de nuevos materiales genéticos estuvo a cargo del Instituto Colombiano Agropecuario (ICA), y a partir de 1993, de la Corporación Colombiana de Investigación Agropecuaria (CORPOICA). Las variedades de la serie Gossica fueron desarrolladas por ICA en las décadas de los setenta y ochenta. CORPOICA ha desarrollado variedades a partir de los cruces entre variedades de alto rendimiento de fibra introducidas de África y variedades colombianas. Estas variedades corresponden a las series de líneas Cesar (LC) y, más recientemente, líneas Cereté (LCER). Ambas series corresponden a variedades convencionales seleccionadas por calidad de fibra requerida por el mercado, estabilidad fenotípica, arquitectura de planta y adaptación específica a los diferentes ambientes ecológicos de las zonas algodoneras colombianas $(8,26)$.

La introducción y el mejoramiento del algodón Upland en Colombia y el mundo han estado determinados por dos criterios básicos: i) rendimiento y calidad de fibra y ii) la resistencia a plagas y enfermedades y la tolerancia a herbicidas. En el caso específico de Colombia, los esfuerzos de mejoramiento se han concentrado básicamente en incrementar la calidad y el rendimiento de fibra, desarrollando variedades con adaptación específica a los nichos ecológicos de las áreas algodoneras del país, buscando satisfacer las necesidades de la industria textil nacional. Para el segundo criterio, los esfuerzos se han concentrado en mejorar los rendimientos de las variedades en el proceso de desmote; las variedades de origen colombiano no han sido seleccionadas específicamente para resistencia a enfermedades o plagas. La incorporación de tecnología transgénica de las variedades estadounidenses alteró el escenario de preferencia de los agricultores, permitiéndoles a estas el dominio del mercado nacional, a expensas de las variedades convencionales. En el caso de las variedades colombianas, toda la información referida a genealogía y características se encuentra en documentos de literatura gris; pero el caso de las variedades introducidas es tal vez menos dramático, pues se encuentran bien descritas (rendimiento y calidad de fibra), así como los transgenes contenidos. En este contexto, los objetivos de esta revisión son: i) describir las características y genealogía de las 
variedades de algodón Upland desarrolladas por los programas de mejoramiento en Colombia y ii) describir las variedades de algodón Upland provenientes de otros países introducidas en el sistema productivo de algodón. El propósito de esta revisión es ofrecer un documento comprensivo sobre la información de cultivares de algodón que son empleados por los agricultores para el establecimiento de las plantaciones. En el futuro, este documento puede ser usado como referencia para programas de mejoramiento genético, incorporación de nuevas variedades o evaluación del impacto del desarrollo e introducción de variedades sobre las brechas de rendimiento.

\section{Introducción de variedades americanas en el sistema productivo colombiano}

\section{A. Características de las variedades americanas de algodón Upland}

La tendencia en los programas de mejoramiento genético de algodón Upland en Estados Unidos ha resultado en incrementos positivos en tres características principales de la fibra: longitud, resistencia y micronaire. Entre 1980 y 2010, el micronaire exhibió una tendencia al aumento, sin que el cambio haya sido realmente significativo. El énfasis del mejoramiento genético en incrementar el rendimiento de fibra ha resultado en el efecto indeseado de fibras con altos valores de micronaire (27-29) y escasa resistencia (30). El incremento de la resistencia de la fibra se debe principalmente al desarrollo del cultivar DP90, en el programa de mejoramiento de New Mexico State University. La longitud de la fibra se incrementó hasta 1997 y declinó hasta 2006, cuando inició el programa de mejoramiento del algodón americano del germoplasma Pee Dee (29). La mayor parte del mejoramiento en fibra de los últimos años puede ser atribuido a la introducción de materiales desarrollados en Australia, principalmente representados por los cultivares de las líneas FiberMax (FM) y DeltaPine (DP). Los análisis de pedigree revelan que DP90 es parte de la base genéticas de las líneas FM y DP, y que gran parte del mejoramiento en calidad de fibra está asociado al material desarrollado en New Mexico State University (31).
La industria textil requiere fibras con diferentes valores de longitud, que correspondan con la calidad del tejido que se va a obtener. Los tejidos de alta calidad requieren fibras finas y largas, y los tejidos de baja calidad y tolerancia al desgaste físico requieren fibras cortas. Con el propósito de atender las necesidades globales del sector textilero, las variedades americanas exhiben un amplio rango de longitudes, de tal manera que pueden ser usadas en una industria textil específica. Las variedades de la serie FiberMax producen fibras de longitudes medias y largas, mientras que las de la serie Stoneville producen fibras de longitud corta $(32,33)$. EI promedio mínimo de la mitad superior (UHM) de la longitud de fibra para los mercados internacionales es $28 \mathrm{~mm}$, pero el mínimo de las variedades estadounidenses es $27 \mathrm{~mm}$. El programa de mejoramiento liderado por Texas A\&M AgriLife Research ha desarrollado líneas de algodón Upland con fibras de mayor longitud y resistencia. Estas líneas se denominan de fibra extralarga, con una longitud UHM mayor a $32 \mathrm{~mm}$, y muchas superan los $34.8 \mathrm{~mm}$, longitud típica de las variedades de algodón Pima (G. barbadense). Las variedades de algodón Upland son preferidas frente a las de algodón Pima, porque exhiben mayores rendimientos (34).

\section{B. Introducción de variedades americanas convencionales}

Las variedades americanas fueron introducidas a principios del siglo pasado y se han constituido en los materiales dominantes del mercado colombiano. Las series americanas DeltaPine, Stoneville y Fibermax conforman este grupo de líneas $(5,13)$. DeltaPine fue introducida al país en la década de los treinta, de manera simultánea con la Stoneville. Casi medio centenar de variedades de estas series han sido introducidas en el país (Suplemento 1). El Instituto de Fomento Algodonero (IFA), creado en 1948, fue el encargado de evaluar un gran número de estos materiales de algodón introducidos en los años cincuenta y sesenta. La incorporación de las variedades americanas marcó el nacimiento de la actual industria algodonera colombiana. Los rendimientos pasaron de $125 \mathrm{~kg}$ fibra/ha a un promedio 
de $1000 \mathrm{~kg}$ fibra/ha, permitiendo el abastecimiento del mercado nacional y la exportación $(5,35)$.

\section{Incorporación de algodones Upland transgénicos}

Los algodones Upland transgénicos resistentes a lepidópteros fue uno de los primeros cultivos modificados genéticamente en ser comercializados; su aprobación para uso comercial en Estados Unidos y Australia se dio en los años 1996-1997 (36), y alcanzó en la primera década del siglo XXI un área sembrada de más de 20 millones de hectáreas (37). Los algodones Upland transgénicos fueron introducidos a Colombia en el 2003, con la tecnología Bollgard ${ }^{\odot}$ de Monsanto. Los cultivares que contienen la proteína Cry1Ac y, posteriormente la proteína Cry2Ab (38), introducidos en Colombia, permitieron reducir a la mitad las aplicaciones para el manejo de insecticidas, y se convirtieron en el material genético dominante en el mercado colombiano y empleado en todas las regiones productoras del país (Tabla 1) (11, 15-18).

Para el año 2010, el área de algodón transgénico en Colombia fue de 37.657 ha, distribuidas entre los departamentos de Córdoba (10.186 ha), Tolima (4.088 ha), Huila (801 ha) y Cesar (3.799 ha) (11). Las tecnologías y genes incorporados en el cultivo de algodón, liberados en Colombia se describen en la Tabla 2. Las proteínas Cry incorporadas en el algodón exhiben actividad biológica general contra varios lepidópteros, especialmente sobre el complejo bellotero (Heliotis virescens Fabricius, Helicoverpa zea Boddie, Spodoptera sp.), el gusano cogollero (Spodoptera frugiperda), el complejo rosado (Pectinophora gossypiella Saunders y Sacadodes pyralis Dyar), el gusano de la hoja del algodonero (Alabama argillacea Hubner), el falso medidor (Trichoplusia ni), el perforador de la hoja (Bucculatrix sp.), el gusano soldado (Spodoptera exigua, Spodoptera ornithogalli) y el gusano defoliador de la hoja (Pseudoplusia includens). En el caso de Spodoptera frugiperda, el control exhibido es parcial y no controla por completo la población, solo afecta el desarrollo y la supervivencia de las larvas (17).

\section{Desarrollo de variedades colombianas de algodón Upland de alto rendimiento y alta calidad de fibra}

Inicialmente, el ICA y, posteriormente, CORPOI$\mathrm{CA}$ se han concentrado en desarrollar variedades de algodón Upland adaptadas a los nichos agroecológicos de las zonas algodoneras colombianas. Los nichos agroecológicos en los cuales se han concentrado los esfuerzos son las regiones denominadas como Caribe Húmedo, Caribe Seco y el Valle Cálido del Alto Magdalena (VCAM). El denominado Caribe Húmedo representa las regiones de monzón (Am, según la clasificación climática Köppen) y el Caribe Seco representa las regiones de sabana (As) de las Planicies del Caribe. EI VCAM, por su parte, incluye la porción más baja del valle superior del Magdalena, ocupando los departamentos de Tolima y Huila. La fracción del Tolima exhibe una tendencia a clima monzónico, mientras la fracción sur exhibe características de sabana (As). Las localidades más representativas de las regiones de monzón de las Planicies del Caribe son el Valle del Sinú, la región productora de algodón más grande del país, y el sur del Cesar, representado por el municipio de Aguachica. Las variedades desarrolladas por ICA y CORPOICA pertenecen a 3 colecciones: 1) Líneas Gossica, 2) Líneas Cesar y 3) Líneas Cereté.

\section{A. Líneas Gossica (GS)}

Las variedades pertenecientes a la serie Gossica fueron desarrolladas por el Instituto Colombiano Agropecuario (ICA) en la década de los setenta, y liberadas a partir de 1977 (Tabla III). Varias de estas líneas fueron usadas como parentales en los programas de mejoramiento de CORPOICA, la institución que absorbió las labores de investigación después de la reforma del ICA en los años noventa. La línea N21 fue incluida en un análisis de estructura genética de poblaciones, revelando que está estrechamente relacionada con la población de la cual se originó la serie TAMCOT y FM (39). 
Tabla III. Descripción de las variedades pertenecientes a la serie Gossica (GS).

\begin{tabular}{|c|c|c|c|c|c|c|c|}
\hline Serie & Código & Variedad & Parenta & & $\begin{array}{c}\text { Año de } \\
\text { liberación } \\
\text { al mercado }\end{array}$ & $\begin{array}{l}\text { Año de } \\
\text { salida del } \\
\text { mercado }\end{array}$ & $\begin{array}{l}\text { Método de } \\
\text { selección }\end{array}$ \\
\hline GS & P11 & Gossica P11 & & & 1984 & 1985 & Selección \\
\hline GS & $\mathrm{P} 12$ & Gossica P12 & $\begin{array}{c}\text { Carolina } \\
\text { Queen }\end{array}$ & Early Staple & 1984 & 1985 & Hibridación \\
\hline GS & $\mathrm{P} 21$ & Gossica P21 & $\begin{array}{r}\text { Acala } 1517 \times \\
\text { BR2 }\end{array}$ & $\begin{array}{l}\text { Stoneville } \\
7 \text { BR }\end{array}$ & 1978 & 1984 & Hibridación \\
\hline GS & $\mathrm{N} 21$ & Gossica N21 & & & 1977 & 1984 & Selección \\
\hline GS & N22 & Gossica N22 & $\begin{array}{r}\text { Acala } 1517 \mathrm{X} \\
\text { BR2 }\end{array}$ & $\begin{array}{l}\text { Stoneville } \\
213\end{array}$ & 1980 & 1982 & Hibridación \\
\hline GS & N23 & Gossica N23 & $\begin{array}{r}\text { Acala } 1517 \\
\text { BR2 }\end{array}$ & $\begin{array}{l}\text { Stoneville } \\
213\end{array}$ & 1983 & 1985 & Hibridación \\
\hline GS & M21 & Gossica M21 & Deltapine $16 \mathrm{X}$ & GRS 684 & 1981 & - & Hibridación \\
\hline GS & MC22 & Gossica MC22 & Deltapine45 A X & $\begin{array}{l}\text { Acala 1517- } \\
70\end{array}$ & - & - & - \\
\hline GS & MC23 & Gossica MC23 & Linea $116 \mathrm{X}$ & $\begin{array}{l}\text { Linea Valle } \\
4\end{array}$ & - & - & Pedigree \\
\hline GS & $\begin{array}{l}\text { MC 23- } \\
828\end{array}$ & $\begin{array}{l}\text { Gossica MC23- } \\
828\end{array}$ & Gossica MC. & $23-828$ & - & - & $\begin{array}{c}\text { Selección de } \\
\text { individuos }\end{array}$ \\
\hline
\end{tabular}

\section{B. Líneas Cesar (LC)}

La serie de Líneas Cesar fueron desarrolladas por CORPOICA a partir de introducciones africanas de alto rendimiento de fibra. A partir de esta colección fueron liberadas al mercado seis nuevas variedades de algodón de fibra media, con adaptación específica a las condiciones agroecológicas de cada región algodonera: Caribeña M129, Corpoica M123 (40, 41), Gaitana M109 (42) y Sinuana M137 (43), (26) (Tabla IV).

Tabla IV. Descripción de las variedades y líneas avanzadas de la serie Líneas Cesar (LC).

\begin{tabular}{|c|c|c|c|c|c|}
\hline \multirow{2}{*}{$\begin{array}{c}\text { Serie } \\
\text { LC }\end{array}$} & \multirow{2}{*}{$\begin{array}{l}\text { Código } \\
\text { LC138 }\end{array}$} & \multicolumn{2}{|c|}{$\begin{array}{l}\text { Variedad / Líneas } \\
\text { experimentales }\end{array}$} & Parentales & \multirow{2}{*}{$\begin{array}{c}\text { Método de } \\
\text { selección } \\
-\end{array}$} \\
\hline & & Línea Cesar & 138 & - & \\
\hline LC & LC137 & Línea Cesar & 137 & STAMF X LC8590 M-9-1-5 & Pedigree \\
\hline LC & LC135 & Línea Cesar & 135 & ISA205A X STV115 M-7-1-9 & Pedigree \\
\hline LC & LC134 & Línea Cesar & 134 & ISA205A X STV115 M-7-1-9 & Pedigree \\
\hline LC & LC129 & Línea Cesar & 129 & ISA205A X STV115 M-2-4-11 & Pedigree \\
\hline LC & LC125 & Línea Cesar & 125 & Gossica-12-1-3 -M-5-5 & - \\
\hline LC & LC124 & Línea Cesar & 124 & ISA205A X STV115 M-2-4-2 & Pedigree \\
\hline LC & LC123 & Línea Cesar & 123 & ISA205A X Gossica-12-1-3)-M-5-5 & Pedigree \\
\hline LC & LC109 & Línea Cesar & 109 & STAM42-A-2-M & - \\
\hline LC & LC107 & Línea Cesar & 107 & ISA205H & - \\
\hline LC & LC137 & Sinuana M & 137 & ISA205A X STV115 M-7-1-9 & Pedigree \\
\hline LC & LC129 & Caribeña $M$ & 129 & ISA205A X STV115 M-2-4-11 & Pedigree \\
\hline
\end{tabular}




\begin{tabular}{ccccccc}
\hline Serie & Código & \multicolumn{2}{c}{$\begin{array}{c}\text { Variedad / Líneas } \\
\text { experimentales }\end{array}$} & \multicolumn{2}{c}{ Parentales } & $\begin{array}{c}\text { Método de } \\
\text { selección }\end{array}$ \\
\hline LC & LC123 & Corpoica M & 123 & ISA205A & X Gossica P-12 1-3-M-3-5 & Pedigree \\
\hline LC & LC110 & Llanera M & 110 & ISA205A & X STV115 M-7-1-7 & Pedigree \\
\hline LC & LC109 & Gaitana M & 109 & ISA205A & X STV115 M-2-4-11 & Pedigree \\
\hline
\end{tabular}

Las líneas LC tienen como parentales a cultivares africanos desarrollados por el CIRAD, líneas de la serie Cossica, STV115 y LC8590 (Tabla V). Los cultivares africanos provenientes del CIRAD corresponden a ISA205A, ISA205H, STAMF y STAM42 (Tabla V). STV115 pertenece a la serie de líneas Stoneville desarrollado en Mississippi. LC8590 es un cultivar colombiano sin registros previos probablemente derivado de algodones silvestres. Los algodones hirsutum colombianos no están emparentados con las poblaciones usados en los programas de mejoramiento de las series STV, TAMCOT y FM (39).

Tabla V. Descripción de los parentales usados en el desarrollo de las líneas LCER.

\begin{tabular}{lllll}
\hline \multirow{2}{*}{ Parentales de la serie LCER } & \multicolumn{3}{c}{ Linaje } & \\
\cline { 2 - 5 } & Cultivar & Origen & Cultivar & Origen \\
\hline LC123 & ISA205A & Malí & x Gossica P12 & Colombia \\
\hline LC129 & ISA205A & Malí & x STV115 & USA \\
LC137 & ISA205A & Malí & x STV 115 & USA \\
Delta Opal* & STAM F & Togo & x LC8590 & Colombia \\
\hline TAMCOT CAND E & USA & & \\
\hline DP5415 & & USA & & \\
\hline DO & & USA & & \\
\hline TAMCOT HQ 95 & USA & & \\
\hline COODETEC401 & USA & & \\
\hline CNPA7H & Brasil & & \\
LC8590 & Brasil & & \\
\hline IRMA1243 & Colombia & & \\
\hline IRMA96+97 & África & & \\
\hline
\end{tabular}

* Números de registro de variedades (PVP) en Estados Unidos: Delta OPAL: 9900136; DP 5415:

9100132; TAMCOT HQ95: 9000092; y TAMCOT CAMD-E: 7800073. Registro PVP para Stoneville 115 no fue encontrado. En multiples documentos, la variedad Delta Opal es denominada DP Opal. Este último no es el nombre oficial.

\section{Líneas Cereté (LCER)}

Las líneas LCER fueron desarrolladas con el propósito de obtener variedades de ciclos vegetativos cortos y compactas con alto rendimiento físico y fibra larga para nichos agroecológicos específicos del país (8); se obtuvieron a partir de 16 cruces (Tabla VI) entre LAMs de la serie LC y cultivares de alto rendimiento de origen americano y brasilero.
A partir de esta colección, han sido liberadas dos nuevas variedades de algodón al mercado del Caribe Húmedo: Corpoica LCER044 (44) y Corpoica LCER007 (45). Las líneas LCER han demostrado mejor desempeño en los siguientes nichos ecológicos del país: Caribe Húmedo, Caribe Seco, Valle Cálido del Alto Magdalena y Altillanura (46). 
Tabla VI. Descripción de los 16 cruces desarrollados para obtener la colección de líneas avanzadas de mejoramiento LCER.

\begin{tabular}{crlll}
\hline Código cruce & & \multicolumn{2}{c}{ Pedigree } & Número de LAMs \\
\hline 1 & LC135 & X & Delta Opal & 9 \\
\hline 2 & LC8590 & X & TAMCOT CAND E & 1 \\
\hline 3 & LC8590 & X & Delta Opal & 13 \\
\hline 4 & IRMA1243 & X & TAMCOT CAB ES & 1 \\
\hline 5 & IRMA1243 & X & IRMA96+97 & 3 \\
\hline 6 & LC123 & X & DP5415 & 1 \\
\hline 7 & LC123 & X & COODETEC401 & 3 \\
\hline 8 & LC129 & X & DP5415 & 7 \\
\hline 9 & LC137 & X & Delta Opal & 2 \\
\hline 10 & LC129 & X & DP5415 & 2 \\
\hline 11 & LC137 & X & Delta Opal & 2 \\
\hline 12 & LC129 & X & Delta Opal & 3 \\
\hline 13 & LC129 & X & DP5415 & 3 \\
\hline 14 & LC137 & X & Delta Opal & 2 \\
\hline 15 & LC137 & X & TAMCOT HQ 95 & 2 \\
\hline 16 & Delta Opal & X & CNPA7H & 4 \\
\hline
\end{tabular}

Adicional al desarrollo de la serie LCER de cultivares, CORPOICA participó en la implementación de un programa de multiplicación y comercialización de semilla y, en el largo plazo, en la incorporación de genes de resistencia a plagas a las variedades nacionales de algodón, en la continua evaluación de variedades introducidas (8) y en el desarrollo de variedades resistentes a limitantes sanitarias. La Tabla VII presenta un resumen de todas las variedades liberadas por ICA-CORPOICA en las últimas dos décadas.

Tabla VII. Descripción de variedades nacionales liberadas en las últimas dos décadas incluyendo rendimientos y nicho ecológico para el cual fueron creadas.

\begin{tabular}{|c|c|c|c|c|c|c|c|c|c|c|c|}
\hline \multirow[t]{2}{*}{ Serie } & Código & Variedad & \multicolumn{2}{|r|}{ Genealogía } & \multirow{2}{*}{$\begin{array}{l}\begin{array}{l}\text { Selecciones } \\
\text { individuales }\end{array} \\
M-6-4-0\end{array}$} & \multirow{2}{*}{$\begin{array}{c}\begin{array}{c}\text { Método de } \\
\text { Mejoramiento }\end{array} \\
\text { Pedigree }\end{array}$} & \multirow{2}{*}{$\begin{array}{l}\text { Creador } \\
\text { ICA - } \\
\text { CORPOICA }\end{array}$} & \multirow{2}{*}{$\begin{array}{l}\text { Adaptación } \\
\begin{array}{l}\text { Valle del } \\
\text { Cauca }\end{array} \\
\end{array}$} & \multirow{2}{*}{$\begin{array}{l}\begin{array}{c}\text { Año de } \\
\text { inscripción }\end{array} \\
1999\end{array}$} & \multirow{2}{*}{$\begin{array}{c}\begin{array}{c}\text { Algodón } \\
\text {-semilla } \\
\mathrm{Kg} / \mathrm{ha}\end{array} \\
2563\end{array}$} & \multirow{2}{*}{$\begin{array}{l}\text { Fibra } \\
\mathrm{Kg} / \mathrm{ha}\end{array}$} \\
\hline & LA1294 & $\begin{array}{l}\text { Delrut } \\
\text { Fibra } \\
\text { Larga }\end{array}$ & LC135 & X Delta Opal & & & & & & & \\
\hline LC & LC110 & $\begin{array}{l}\text { Llanera } \\
\text { M110 }\end{array}$ & $\begin{array}{l}\text { ISA } 205 \\
\text { A }\end{array}$ & X STV 115 & $M-7-1-7$ & Pedigree & $\begin{array}{l}\text { ICA - } \\
\text { CORPOICA }\end{array}$ & $\begin{array}{l}\text { Llanos } \\
\text { Orientales }\end{array}$ & 2000 & 2302 & 1005 \\
\hline GS & MC23 & $\begin{array}{l}\text { Gossica } \\
\text { MC23 }\end{array}$ & LC135 & X Delta Opal & M-6-4-1 & Pedigree & ICA & $\begin{array}{l}\text { Llanos } \\
\text { Orientales }\end{array}$ & 2000 & 2623 & 1087 \\
\hline \multirow{3}{*}{ LC } & \multirow{3}{*}{ LC129 } & \multirow{3}{*}{$\begin{array}{l}\text { Caribeña } \\
\text { M129 }\end{array}$} & \multirow{3}{*}{$\begin{array}{l}\text { ISA } \\
205 A\end{array}$} & \multirow{3}{*}{$\begin{array}{l}\text { Stoneville } \\
115\end{array}$} & \multirow{3}{*}{$M-2-4-11$} & \multirow{3}{*}{ Pedigree } & \multirow{3}{*}{$\begin{array}{l}\text { ICA - } \\
\text { CORPOICA }\end{array}$} & $\begin{array}{l}\text { Caribe } \\
\text { Seco }\end{array}$ & \multirow{3}{*}{2001} & 2893 & 1229 \\
\hline & & & & & & & & $\begin{array}{l}\text { Valle del } \\
\text { Cauca }\end{array}$ & & 2388 & 1092 \\
\hline & & & & & & & & $\begin{array}{l}\text { Llanos } \\
\text { Orientales }\end{array}$ & & 2298 & 1005 \\
\hline
\end{tabular}




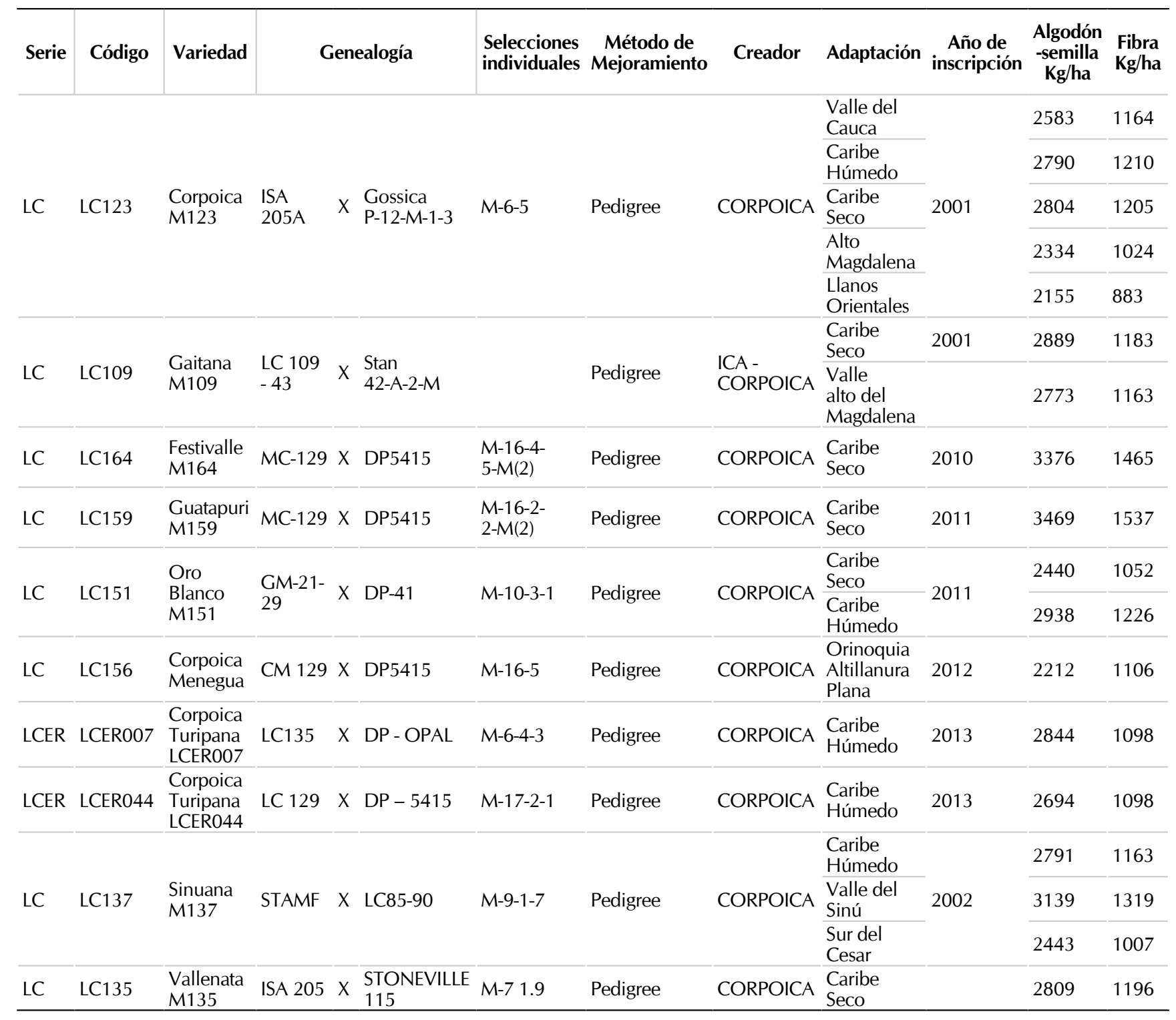

\section{Nuevas perspectivas de variedades de algodón}

En el escenario actual, los esfuerzos deben concentrarse en el incremento del rendimiento, especialmente en las regiones de monzón y sabana, donde se registran los rendimientos más bajos, y en el desarrollo de variedades resistentes a plagas y enfermedades. Adicionalmente, es necesario desarrollar cultivos transgénicos de algodón con base en las variedades nacionales; esto permite la adquisición de características que no pueden ser incorporadas en los materiales nacionales a través del mejoramiento tradicional. En este aspecto, es importante la incorporación de cultivares transgénicos resistentes al picudo del algodón, la principal limitante sanitaria para los cultivos de algodón en el país. Los siguientes párrafos describen los esfuerzos recientes en mejoramiento de algodón en Colombia.

\section{A. Incorporación de genes transgénicos en materiales colombianos de alto rendimiento}

En la última década, los esfuerzos de CORPOICA se han concentrado en la incorporación de los ge- 
nes cry $1 A c$, cry2Ab y cp4 epsps en variedades seleccionadas de las líneas LC y LCER. Estos nuevos materiales exhibirán altos rendimientos y longitud de fibra media. En el caso de las líneas LC serán los primeros materiales transgénicos de algodón adaptados a las condiciones del trópico de monzón y sabana.

\section{B. Desarrollo de variedades resistentes a limitantes sanitarias}

El síndrome de ramulosis del algodón ha sido recientemente reportado para Colombia (47), pero probablemente ha estado presente en el país por más de una década; esta enfermedad es favorecida, principalmente, por las precipitaciones constantes durante el ciclo de cultivo $(48,49)$. La ramulosis es la principal enfermedad de las zonas algodoneras colombianas, y es especialmente importante en el Valle del Sinú. Este valle tiene un clima monzónico, bajo el cual los cultivos son establecidos durante la temporada lluviosa, incrementando el riesgo de desarrollo de la enfermedad (49). Las pérdidas son marginales para los agricultores industriales, pero limitantes en los agricultores pequeños. Evaluaciones en campo de los materiales LCER revelan que existen por lo menos dos fuentes de resistencia a la ramulosis, presentes en una decena de LAMs que pueden ser liberadas como cultivares o introducidas en los programas de mejoramiento.

\section{Estado actual del desarrollo de resistencia a Anthonomus grandis}

Anthonomus grandis (Coleoptera: Curculionidae), o el picudo del algodón, es la plaga más limitante del cultivo en las Américas. En Colombia representa el principal problema sanitario, y la estrategia de manejo puede superar el $10 \%$ de los costos de producción (50). En la región Caribe colombiana han sido necesarias hasta 15 aplicaciones por ciclo (51). Estos tratamientos son necesarios porque los adultos se alimentan exclusivamente de las estructuras terminales tiernas, del polen de las flores y de los frutos (botones florales y cápsulas). Las larvas están protegidas en el interior de las cápsulas o en los botones florales, lo cual dificulta el contacto con los insecticidas químicos. Sin embargo, el control de esta plaga, usando múltiples aplicaciones, es la única estrategia (inefectiva) de manejo de esta plaga.

Existe una alternativa de solución para este problema: el uso de plantas transgénicas de algodón con resistencia a $A$. grandis. La mayor parte de las proteínas disponibles para control de plagas provienen de Bacillus subtilis, denominadas Cry. La inserción del gen cry en el genoma de la planta permite la producción de una proteína cristalina inocua para la planta, pero tóxica para los insectos. Las toxinas Cry son efectivas contra lepidópteros, coleópteros y dípteros (52). El uso de estas toxinas no solo disminuye los costos de manejo e incrementa la productividad, sino que ambientalmente son amigables, al reducir la cantidad de pesticidas requeridos y, en consecuencia, disminuir los efectos nocivos de la agricultura industrial sobre los trabajadores rurales y el ambiente (53).

En el caso del algodón, los genes cry insertados permiten el control de lepidópteros (Tabla I) y, junto con los genes de tolerancia a herbicidas, son las principales características de las variedades de algodón transgénicas comerciales. Ninguna de las variedades transgénicas comerciales contiene genes de resistencia a $A$. grandis o $S$. frugiperda; sin embargo, varias proteínas Cry exhiben actividad biológica contra estas plagas (Tabla I) y al menos una de ellas, la proteína Cry1la12, ha sido insertada en plantas de algodón, confiriendo resistencia parcial a estas plagas (54-56). La incorporación de este tipo de algodón transgénico, que contiene el gen cry1la12, reduciría los costos de manejo de plagas en el Valle del Sinú alrededor de la mitad del valor actual, con una reducción en costos totales del $7 \%$. Es bastante probable que la incorporación de esta proteína provoque un incremento significativo en el rendimiento (51).

Recientemente han sido liberados en el mercado estadounidense materiales de algodón que contienen la tecnología Bollgard 3, que incluye las 2 proteínas Cry ya presentes en los materiales transgénicos (Cry1Ac y Cry2Ab) y una tercera proteína, la Vip3A. Esta tecnología ha sido desarrollada para disminuir la probabilidad de resistencia que los lepidópteros pueden desarrollar, especialmente la resistencia de Helicoverpa spp. a las proteínas $\mathrm{Bt}$ 
(57). No hay ninguna evidencia de que esta proteína tiene actividad alguna contra el picudo del algodón, así que la incorporación de esta tecnología en el sistema productivo del algodón solo traerá un efecto marginal sobre el control de Spodoptera.

Esta revisión compiló material bibliográfico catalogado como literatura gris para ofrecer una visión actualizada de los materiales de algodón incorporados en el sistema productivo colombiano. Es de especial importancia la inclusión de información consolidada sobre la generación de variedades de algodón nacionales desarrolladas por el ICA y CORPOICA, especialmente la genealogía de estas variedades. Un documento adicional en desarrollo se encargará de describir las ganancias de rendimiento, calidad de fibra y resistencia a enfermedades de estos programas de mejoramiento.

\section{Perspectivas}

Finalmente, en una perspectiva de las variedades nacionales, es necesario considerar los niveles de adopción de estas variedades y la mejor estrategia de mejoramiento. La adopción de las variedades nacionales requiere ser analizada considerando porcentajes de las áreas sembradas, rendimientos, producción, costo de la semilla, cambios en los sistemas de siembra (siembra manual contra siembra de precisión) y las ventajas y problemas de estas variedades en la producción de algodón de fibra media en Colombia.

En el caso de los programas nacionales de mejoramiento genético, es necesario evaluar el desarrollo de alianzas estratégicas con multinacionales o instituciones de investigación internacionales, en procura de cerrar las brechas tecnológicas hoy existentes. Un programa de mejoramiento de este tipo permitiría incrementar las probabilidades de éxito, sostenibilidad, competitividad y equidad, además de reducir costos y tiempo y racionalizar recursos e infraestructura.

La brecha tecnológica es en este momento mucho más amplia con el desarrollo de la tecnología CRISPR/Cas9, que permite la edición de genomas con alta eficiencia y especificidad (58-63). El algodón Upland presenta múltiples problemas de mejoramiento en consideración a la naturaleza tetraploide del genoma; sin embargo, todas estas limitaciones pueden ser superadas con el uso de CRISPR/Cas9. En el momento, un sistema eficiente de manipulación genética, basado en esta tecnología, ha sido desarrollado para el algodón Upland, el cual tiene como propósito la modificación de los genes implicados en la biosíntesis de gossypol, la regulación negativa del desarrollo de la fibra y la resistencia a enfermedades $(64,65)$.

\section{Conclusiones}

Las variedades de algodón extranjeras han estado dominadas por los materiales de origen americano, que en la última década son de tipo transgénico; los genes que contienen estos materiales les confieren resistencia a lepidópteros y tolerancia a herbicidas. Las variedades nacionales hasta el momento liberadas son del tipo convencional y pertenecen a tres programas de mejoramiento (series), identificados como GS, LC y LCER; estos materiales están adaptados para exhibir mayores rendimientos y mejor calidad de fibra (longitud, resistencia y micronaire) en los diferentes nichos ecológicos donde se produce algodón. La serie LCER ha sido diseñada particularmente para adaptación a los climas de sabana y monzón predominantes en la región Caribe; esta región es precisamente la que exhibe las mayores brechas de rendimiento en el país.

\section{Financiación y afiliación de los autores}

Esta investigación fue desarrollada dentro del marco del Macroproyecto "Generación de variedades y desarrollo de recomendaciones tecnológicas para los sistemas de producción de los cultivos de algodón, arroz y maíz en los Valles interandinos, Caribe Colombiano y piedemonte Ilanero", el cual fue financiado con fondos del Ministerio de Agricultura y Desarrollo Rural (C09515). Por su parte, la Red de Cultivos Transitorios y Agroindustriales - CORPOICA brindo el apoyo logístico. Oscar Burbano-Figueroa (OBF) y Karen Sofia Montes-Mercado (KSMM) colectaron datos y escribieron el manuscrito. OBF y KSMM pertenecen a The Plant Interactions Laboratory, Centro de Investigación Turipaná, Red de Cultivos Transitorios y Agroindustriales, CORPOICA (Cereté, Córdoba, Colom- 
bia). Ivan Javier Pastrana-Vargas (IJPV) pertenece al Centro de Investigación Motilonia, Red de Cultivos Transitorios y Agroindustriales, CORPOICA (Agustín Codazzi, Cesar, Colombia). IJPV y JCT revisaron el manuscrito y suministraron los datos a esta revisión.

\section{Referencias}

(1) Gotmare V. Wild and Cultivated Species of Cotton. Singh P, Tule BN, editors. Nagpur: Central Institute for Cotton Research; 2011.

(2) Paterson AH., Wendel JF., Gundlach H., Guo H., Jenkins J., Jin D., et al. Repeated polyploidization of Gossypium genomes and the evolution of spinnable cotton fibres. Nature. 2012; 492(7429): 423-437. DOI: http://doi.org/10.1038/nature11798.

(3) Li F., Fan G., Lu C., Xiao G., Zou C., Kohel RJ., et al. Genome sequence of cultivated Upland cotton (Gossypium hirsutum TM-1) provides insights into genome evolution. Nat Biotechnol. 2015; 33(5): 524-30. DOI: http://doi.org/10.1038/nbt.3208.

(4) Wendel JF. New World tetraploid cottons contain Old World cytoplasm. Proc Natl Acad Sci USA. 1989; 86(11): 4132-4136. DOI: http://doi.org/10.1073/pnas.86.11.4132.

(5) Leurquin P. Cotton growing in Colombia: achievements and uncertainties. Food Research Institute; 1966.

(6) Brochero MF. Importancia socioeconómica del cultivo del algodón en Colombia. La producción de semillas de algodón para siembra en Colombia. Bogotá: Instituto Colombiano Agropecuario (ICA); 1987.

(7) Espinal CF., Martínez Covaleda H., Pinzón Ruíz N., Barrios Urrutia C. La cadena de algodón en Colombia: una mirada global de su estructura y dinámica 1991-2005 [Internet]. Bogota: Banco de la República; 2005 [cited 2018 Jan 18]. Disponible en: http:// bibliotecadigital.agronet.gov.co/handle/11348/6113.

(8) Cadena Torres J. Organización de la investigación en algodón en Colombia. En: XI Reunión Asociación Latinoamericana de Investigación y Desarrollo del Algodón. Argentina: ALIDA; 2010.

(9) Conalgodón. Área, producción y rendimiento. Bogotá: Conalgodón; 2017.

(10) Barragán Quijano E. Determinación de zonas óptimas para la producción del algodón (Gossypium hirsutum I.) en el valle cálido del Alto Magdalena por ambiente, rendimiento y calidad de fibra con énfasis en potasio [Doctoral dissertation]. Universidad Nacional de Colombia; 2010.

(11) Rache Cardenal LY. Monitoreo del flujo de genes de algodón transgénico en la Agremiación Remolino S.A. (Espinal-Tolima) [Tesis de maestría]. Universidad Nacional de Colombia; 2011.

(12) IICA, MADR, CORPOICA. Competitividad de la producción algodón: Proyecto de análisis de competitividad del sector agropecuario colombiano. 1995.

(13) Porter HG. The cotton industry of Colombia [Internet]. Washington D.C.: Foreign Agricultural Service USDA; 1961 [fecha de acceso: 14 de enero de 2018]. Disponible en: http://archive.org/ details/cottonindustryof113port.
(14) Barragán E., Díaz A., Caicedo A., Montenegro O., Mendoza A. Módulo 2: El algodonero - Manejo integrado del cultivo en el VaIle Cálido del Alto Magdalena. El algodonero - Manejo integrado del cultivo en Colombia. Bogotá: Corpoica; 2005.

(15) Amaya OS., Restrepo OD., Argüelles J., Garramuño EA. Evaluación del comportamiento del complejo Spodoptera con la introducción de algodón transgénico al Tolima, Colombia. Revista Corpoica Ciencia y Tecnología Agropecuaria. 2013; 10(1): 2432. DOI: http://doi.org/10.21930/rcta.vol10_num1_art:125.

(16) Valencia SJ. Efecto de las tecnologías Bollgard ${ }^{\circledR}$ y Bollgard II ${ }^{\circledR}$ de variedades genéticamente modificadas (GM) de algodón sobre larvas y adultos del falso bellotero Spodoptera frugiperda Smith (Lepidoptera: Noctuidae) [Undergraduate thesis]. Universidad Nacional de Colombia; 2013.

(17) Valencia Cataño SJ., Rodríguez Chalarca J., Mesa Cobo NC. Efecto de variedades de algodón genéticamente modificadas sobre larvas de Spodoptera frugiperda Smith (Lepidoptera: Noctuidae). Acta Agron. 2014; 63(1): 63-70. DOI: http://doi.org/10.15446/ acag.v63n1.38356.

(18) Giraldo AC., Rache Cardenal LY., Oberlaender JM. Study of gene flow from GM cotton (Gossypium hirsutum) varieties in El Espinal (Tolima, Colombia). Acta Biológica Colombiana. 2013; 18 (3): 489-498

(19) Blanco Martínez J., Rache Cardenal L., Alfonso N., Chaparro Giraldo A. Convenio interadministrativo de asociación suscrito entre el Ministerio de Ambiente, Vivienda y Desarrollo Territorial y la Universidad Nacional de Colombia. Bogotá: Conalgodón; 2011.

(20) Akhurst RJ., James W., Bird LJ., Beard C. Resistance to the Cry1Ac -endotoxin of Bacillus thuringiensis in the cotton bollworm, Helicoverpa armigera (Lepidoptera: Noctuidae). J Econ Entomol. 2003; 96 (4):1290-1299. DOI: http://doi.org/10.1093/ jee/96.4.1290.

(21) Sivasupramaniam S., Moar WJ., Ruschke LG., Osborn JA., Jiang C., Sebaugh JL., et al. Toxicity and characterization of cotton expressing Bacillus thuringiensis Cry1 Ac and Cry2Ab2 proteins for control of lepidopteran pests. Journal of Economic Entomology. 2008; 101(2): 546-54. DOI: http://doi.org/10.1093/ jee/101.2.546.

(22) Adamczyk Jr JJ., Gore J. Laboratory and field performance of cotton containing Cry1ac, Cry1f, and both Cry1ac and Cry $1 \mathrm{f}$ (widestrike ${ }^{\circledR}$ ) against beet armyworm and fall armyworm larvae (Lepidoptera: Noctuidae). Florida Entomologist. 2004; 87(4): 427-432. DOI: http://doi.org/10.1653/0015-4040(2004)087[042 7:LAFPOC]2.0.CO;2.

(23) Sweeney JA., Jones MA. Glufosinate Tolerance of Multiple WideStrike and Liberty-Link Cotton (L.) Cultivars. Crop Sci. 2015; 55(1): 403. DOI: http://doi.org/10.2135/cropsci2014.03.0175.

(24) Kurtz RW., McCaffery A., O'Reilly D. Insect resistance management for Syngenta's VipCotTM transgenic cotton. Journal of invertebrate pathology. 2007; 95(3): 227-230. DOI: http://doi. org/10.1016/j.jip.2007.03.014.

(25) Funke T., Han H., Healy-Fried ML., Fischer M., Schönbrunn E. Molecular basis for the herbicide resistance of Roundup Ready 
crops. Proc Natl Acad Sci USA. 2006; 103(35): 13010-13015. DOI: http://doi.org/10.1073/pnas.0603638103.

(26) Barragán Quijano E., Cadena Torres J. Mejoramiento genético y avances en biotecnología del algodón en Colombia. En: ALIDA. Chaco, Argentina; 2010.

(27) Campbell BT., Chee PW., Lubbers E., Bowman DT., Meredith WR., Johnson J., et al. Dissecting Genotype $\times$ Environment Interactions and Trait Correlations Present in the Pee Dee Cotton Germplasm Collection following Seventy Years of Plant Breeding. Crop Sci. 2012; 52(2): 690. DOI: http://doi.org/10.2135/ cropsci2011.07.0380.

(28) Campbell BT., Chee PW., Lubbers E., Bowman DT., Meredith WR., Johnson J., et al. Genetic Improvement of the Pee Dee Cotton Germplasm Collection following Seventy Years of Plant Breeding. Crop Sci. 2011; 51(3): 955. DOI: http://doi. org/10.2135/cropsci2010.09.0545.

(29) Campbell BT., Weaver DB., Sharpe R., Wu J., Jones DC. Breeding potential of elite pee dee germplasm in upland cotton breeding programs. Crop Sci. 2013; 53: 894-905. DOI: http://doi. org/10.2135/cropsci2012.09.0549.

(30) Clement JD., Constable GA., Stiller WN., Liu SM. Negative associations still exist between yield and fibre quality in cotton breeding programs in Australia and USA. Field Crops Res. 2012; 128: 1-7. DOI: http://doi.org/10.1016/j.fcr.2011.12.002.

(31) Kuraparthy V., Bowman DT., Kuraparthy V., Bowman D. Gains in breeding Upland cotton for fiber quality. J Cotton Sci. 2013; 17: 157-162.

(32) Bayer Colombia. El algodón es una materia prima muy codiciada, aunque su cultivo no resulta fácil. Crop Science Colombia [Internet]. 2015 [fecha de acceso: 27 de junio 2017]. Disponible en: http://www.cropscience.bayer.co/es-CO/Centro-de-Noticias/ Noticias/2015/01/En_estrecho_contacto_con_las_fibras.aspx?overviewld = 7CCOF601-0F91-41AB-89E4-6998110C8286.

(33) Nichols S., Snipes C., Jones M. Cotton growth, lint yield, and fiber quality as affected by row spacing and cultivar. The Journal of Cotton Science. 2004; 8: 1-12.

(34) Smith CW., Hague S., Hequet E., Thaxton PS., Brown IN. Development of Extra-Long Staple Upland Cotton. Crop Sci. 2008; 48(5): 1823. DOI: http://doi.org/10.2135/cropsci2008.01.0052.

(35) Arteta Hernández CM. Una experiencia inconclusa. El cultivo de algodón en el departamento del Atlántico, 1935-1965. Historia Caribe. 2012.

(36) ICAC. Bollgard II: comercialización de nueva generación de genes Bt. ICAC Recorder. 2003.

(37) James C. Global Status of Commercialized Biotech/GM Crops. International Service for the Acquisition of Agri-biotech Applications, Ithaca, NY; 2014.

(38) ICA. Resolución N. ${ }^{\circ} 0310$ (feb. 11/2008). 2008.

(39) Hinze LL., Hulse-Kemp AM., Wilson IW., Zhu Q-H., Llewellyn DJ., Taylor JM., et al. Diversity analysis of cotton (Gossypium hirsutum L.) germplasm using the CottonSNP63K Array. BMC Plant Biol. 2017 Feb 3; 17(1): 37. DOI: http://doi.org/10.1186/ s12870-017-0981-y.
(40) Urrea Gómez R., Garcés Gutiérrez R., Aramendiz Tatis H., Mendoza Olivella A. Nuevas variedades de algodón adaptadas al Valle del Sinú. En: CORPOICA, CONALGODON (eds.). Aportes tecnólogicos a la producción competitivia y sostenible del algodonero en la región Caribe. 2000.

(41) Cadena Torres J., Urrea Gómez R., Mendoza Ollivella A., Jiménez Mass N., Murillo Solano J., Negrete Barón F., et al. Corpoica M-123 Nueva variedad de algodón de fibra media con adaptación al Caribe Húmedo. CORPOICA (ed.), Plan Nacional de Algodón. Cereté: CORPOICA; 2001. Report N. ${ }^{\circ} 1$.

(42) Gaitana M. Variedad rendidora de excelente calidad textil apta para las zonas secas del alto Magdalena. Corpoica.

(43) Corpoica. Variedad de algodón Sinuana M-137, adaptada a las condiciones de la subregión del Caribe Húmedo. Valle del Sinú, las Sabanas de Sucre y Bolívar y el Bajo Magdalena [Internet]. Bogotá: CORPOICA; 2017 [acceso: 22 de junio de 2017]. Disponible en: http://corpoica.org.co/menu/ ot/cultivos-transitorios-y-agroindustriales/170-variedad-de-algod $\%$ C $3 \%$ B 3n-sinuana-m-137-adaptada-a-las-condiciones-de-la-subregi \% C3\%B3n-del-caribe-h\%C3\%BAmedo-vaIle-del-sin \% C3\% BA-las-sabanas-de-sucre-y-bol\%C3\% ADvar-y-el-bajo-magdalena/.

(44) Cadena Torres J., Burbano Figueroa O., Agámez Saibis A., Espitia Camacho M., Campo Arana R. Corpoica Turipaná LCER0044.

(45) Cadena Torres J., Burbano Figueroa O., Agámez Saibis A., Espitia Camacho M., Campo Arana R. Corpoica Turipaná LCER0007.

(46) Campuzano-Duque LF., Caicedo-Guerrero S., Guevara-Agudelo J. Determinación de atributos en genotipos de algodón (Gossypium hirsutum L.) en la rotación maíz-soya asociados a suelos ácidos mejorados de la altillanura colombiana. Corpoica. 2015.

(47) Moreno-Morán M., Burbano-Figueroa O. First report of Colletotrichum gossypii var. cephalosporioides causing cotton ramulosis in Colombia. Plant Dis. 2016; 100(3): 653. DOI: http://doi. org/10.1094/PDIS-08-15-0933-PDN.

(48) Monteiro JEBA., Sentelhas PC., Gleason ML., Esker PD., Chiavegato EJ. Development of ramulosis disease of cotton under controlled environment and field conditions. Phytopathology. 2009; 99(6): 659-665. DOI: http://doi.org/10.1094/PHYTO-99-6-0659.

(49) Moreno-Morán M., Burbano-Figueroa O. Dynamics of cotton ramulosis epidemics caused by Colletotrichum gossypii var. cephalosporioides in Colombia. Eur J Plant Pathol. 2017; 12: 1-12. DOI: http://doi.org/10.1007/s10658-017-1194-9.

(50) Zambrano P., Fonseca LA., Cardona I., Magallanes E. The socio-economic impact of transgenic cotton in Colombia. In: Tripp $\mathrm{R}$ (ed.). Biotechnology and agricultural development: Transgenic cotton, rural institutions and resource poor farmers. London: CRC Press; 2009.

(51) Osorio-Almanza L., Burbano-Figueroa O., Martínez-Reina A. Evaluación de la factibilidad técnica de variedades de algodón expresando proteínas Cry tóxicas contra Anthonomus grandis en el cultivo de algodón en el Valle del Sinú, Colombia. (En prensa). 2018. 
(52) Bravo A., Soberón M. How to cope with insect resistance to Bt toxins? Trends Biotechnol. 2008; 26(10): 573-579. DOI: http:// doi.org/10.1016/j.tibtech.2008.06.005.

(53) ISAAA. Global Status of Commercialized Biotech/GM Crops: 2016. Ithaca, NY: International Service for the Acquisition of Agr-biotech Applications (ISAAA); 2016.

(54) de Oliveira RS., Oliveira-Neto OB., Moura HFN., de Macedo LLP., Arraes FBM., Lucena WA., et al. Transgenic Cotton Plants Expressing Cry1la12 Toxin Confer Resistance to Fall Armyworm (Spodoptera frugiperda) and Cotton Boll Weevil (Anthonomus grandis). Front Plant Sci. 2016; 7: 165. DOI: http://doi. org/10.3389/fpls.2016.00165.

(55) Silva CR., Monnerat R., Lima LM., Martins ÉS., Melo Filho PA., Pinheiro MP., et al. Stable integration and expression of a cry1 la gene conferring resistance to fall armyworm and boll weevil in cotton plants. Pest Manag Sci. 2016; 72(8): 1549-1557. DOI: http://doi.org/10.1002/ps.4184.

(56) Ribeiro TP., Arraes FBM., Lourenço-Tessutti IT., Silva MS., Lisei-de-Sá ME., Lucena WA., et al. Transgenic cotton expressing Cry10Aa toxin confers high resistance to the cotton boll weevil. Plant Biotechnol J. 2017; 15(8): 997-1009. DOI: https://doi. org/10.1111/pbi.12694.

(57) Monsanto. Monsanto | Bollgard 3 [Internet]. 2017 [fecha de acceso: 29 de sep. de 2017]. Disponible en: http://www.monsantoglobal.com/global/au/products/pages/bollgard3.aspx.

(58) Liu D., Hu R., Palla KJ., Tuskan GA., Yang X. Advances and perspectives on the use of CRISPR/Cas9 systems in plant genomics research. Curr Opin Plant Biol. 2016; 30: 70-77. DOI: http://doi. org/10.1016/j.pbi.2016.01.007.

(59) Schaeffer SM., Nakata PA. CRISPR/Cas9-mediated genome editing and gene replacement in plants: Transitioning from lab to field. Plant Sci. 2015; 240: 130-142. DOI: http://doi. org/10.1016/j.plantsci.2015.09.011.

(60) Kumar V., Jain M. The CRISPR-Cas system for plant genome editing: advances and opportunities. J Exp Bot. 2015; 66(1): 47-57. DOI: http://doi.org/10.1093/jxb/eru429.

(61) Bortesi L., Fischer R. The CRISPR/Cas9 system for plant genome editing and beyond. Biotechnol Adv. 2015; 33(1): 41-52. DOI: http://doi.org/10.1016/j.biotechadv.2014.12.006.

(62) Belhaj K., Chaparro-García A., Kamoun S., Nekrasov V. Plant genome editing made easy: targeted mutagenesis in model and crop plants using the CRISPR/Cas system. Plant Methods. 2013; 9(1): 39. DOI: http://doi.org/10.1186/1746-4811-9-39.

(63) Hsu PD., Lander ES., Zhang F. Development and applications of CRISPR-Cas9 for genome engineering. Cell. 2014; 157(6): 12621278. DOI: http://doi.org/10.1016/j.cell.2014.05.010.

(64) Li C., Unver T., Zhang B. A high-efficiency CRISPR/Cas9 system for targeted mutagenesis in Cotton (Gossypium hirsutum L.). Sci Rep. 2017; 7: 43902. DOI: http://doi.org/10.1038/srep43902.

(65) Gao W., Long L., Tian X., Xu F., Liu J., Singh PK., et al. Genome Editing in Cotton with the CRISPR/Cas9 System. Front Plant Sci. 2017; 8:13-64. DOI: http://doi.org/10.3389/fpls.2017.01364. 